\title{
Rasgos Tributarios en los Comerciantes de la Festividad del Corpus Christi
}

\section{Tax Features in Traders of the Corpus Christi Festivity}

INFORMACIÓN DEL

\section{ARTÍCULO}

Fecha de recepción: 13 de Septiembre de 2019.

Fecha de aceptación: 22 de Mayo de 2020

${ }^{I}$ Magíster en Contabilidad y Finanzas, Universidad Espíritu Santo. Docente e Investigador de la Universidad Católica de Cuenca-Ecuador.

E-mail: pmendietaa@ucacue.edu.ec Código ORCID:

https://orcid.org/0000-0001-9596-4344

\section{Patricio Esteban Mendieta Andrade ${ }^{1}$}

\section{Resumen}

En vista de la importancia que tiene la recaudación fiscal como eje del Presupuesto Nacional y como factor redistributivo de la riqueza de la sociedad, esta investigación tiene como propósito reconocer las percepciones y actitudes de los comerciantes de dulces de la festividad del Corpus Christi efectuada en el año 2018 en la ciudad de Cuenca, Ecuador; constituyéndose este trabajo como un instrumento de evaluación del Régimen Tributario Ecuatoriano y su principio de simplicidad administrativa. Se aplicó una investigación exploratoria que involucró a 113 comerciantes; para el análisis de los datos se utilizó estadística descriptiva, la prueba de Chi-cuadrado y el Coeficiente de Correlación de Spearman. Se concluye que RISE es un modelo de tributación favorable a la formalización de los emprendedores, advirtiendo señas del limitado favor que hace el uso de las herramientas informáticas en el cumplimiento de los deberes fiscales por parte de los contribuyentes.

\section{Palabras Clave:}

Corpus Christi, tributación, simplicidad administrativa, fiscalidad, RISE, emprendimiento.

Clasificación JEL: H21, K32, L26.

\begin{abstract}
In view of the importance of tax collection as the axis of the National Budget and as a redistributive factor of the wealth of our society, the purpose of this research is to recognize the perceptions and attitudes of the candy merchants of the Corpus Christi festivity carried out in 2018 in the city of Cuenca, Ecuador; The present work is constituted as an instrument for evaluating the Ecuadorian Tax Regime and its principle of administrative simplicity. Methodologically, information was collected through surveys. An exploratory investigation was applied that involved a sample of 113 merchants; which was analyzed under the parameters of for the analysis of the data, descriptive statistics were used, and the use of the Chi-square test and the Spearman Correlation Coefficient,. It is concluded that recognizing the RISE as it is a tax model favorable to the formalization of entrepreneurs, noting signs of the limited favor that the use of computer tools does in the fulfillment of tax duties by taxpayers.
\end{abstract}

http://dx.doi.org/10.31095/podium.202 0.37 .1

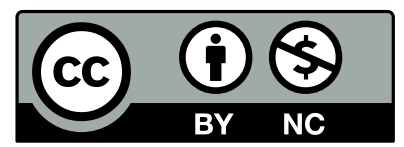

\section{Keywords:}

Corpus Christi, taxation, administrative simplicity, taxation, RISE, entrepreneurship.

JEL Classification: H21, K32, L26. 


\section{Introducción}

La ciudad de Cuenca, capital de la provincia de Azuay, ubicada al sur de los Andes ecuatorianos, hoy en día es reconocida por sus diversas tradiciones, las cuales dan cuenta de sus profundos valores culturales de carácter religioso de origen español, mismas que son preservadas tanto por sus autoridades y conciudadanos, destacándose, dentro de éstas, las festividades del Pase del Niño Viajero, la celebración del Viernes Santo y el Corpus Christi, cada una acompañada con sus correspondientes comidas y bebidas típicas, como la chicha de jora, la fanesca y los tradicionales dulces de Corpus, respectivamente, que hacen a la ciudad merecedora de diferentes galardones tanto en el orden nacional como internacional (Prada, Armijos, Creso, \& Torres, 2018).

Al remontarnos al origen del festejo clerical, Bermejo (2012) reconoce a Santa Juliana de Mont Cornillon en la ciudad de Lieja, Bélgica, en el año 1246, como promotora de la celebración del Corpus Christi, como la exaltación de la presencia Cristo resucitado en el rito eucarístico (Viera y Clavijo, 2017). Se reconoce al año 1557 como la fecha en la que el Cabildo cuencano reconoce e instaura la festividad, desarrollándose en paralelo la tradición de las ventas de dulces en la Plaza Central, actividad realizada por las mujeres conocedoras del oficio traído desde Europa (Fundación Municipal de Turismo de Cuenca, 2015). De forma particular el Corpus Christi o también llamada Fiesta de la Eucaristía, se celebra en Cuenca durante siete días por lo que el festejo adopta el nombre del Septenario; donde, hasta la actualidad se dan cita comerciantes de los tradicionales dulces, constituyéndose en una actividad tanto cultural como económica (Astudillo, 2011).

Subsecuente a lo señalado, se distingue que en el marco de formalización de las actividades económicas, surgen las diversas obligaciones tributarias de los comerciantes, realidad que se enmarca en la importancia que tienen los impuestos en la economía nacional ecuatoriana; dado que, éstos representaron el $44 \%$ del total de los ingresos mediante los cuales el estado presupuestó financiar su gasto en el año 2019 (Ministerio de Economía y Finanzas, 2018); siendo que de ésta manera, se consolida como fundamental el cumplimento del Marco Constitucional Ecuatoriano, por el cual se establece que el Régimen Tributario Ecuatoriano se regirá por los principios de generalidad, progresividad, simplicidad administrativa entre otros; especificándose el papel de la política tributaria como eje promotor de la redistribución y estímulo del empleo, la producción y conductas ecológicas, sociales y económicas responsables (Asamblea Constituyente, 2008). Es así que en este Marco Legal se originan las diferentes obligaciones tributarias como vínculo jurídico entre el Estado y los contribuyentes; las cuales, nacen cuando se realiza el presupuesto establecido por la ley para configurar el tributo (Congreso Nacional, 2005); de ésta manera el Servicio de Rentas Internas (SRI), creado en el año 1997, cumple su 
papel como entidad técnica y autónoma con su facultad, entre otras, de ejecutar la política tributaria, ya anotada (Congreso Nacional, 1997).

En este contexto, con el afán de facilitar el cumplimiento de los tributos por parte de los sectores informales, en el año 2008 mediante la Ley Reformatoria para la Equidad Tributaria se implementa en nuestro país el Régimen Impositivo Simplificado (RISE), como un modelo fiscal de inscripción voluntaria que substituye a la declaración y pago tanto del Impuesto al Valor Agregado (IVA) como al Impuesto a la Renta (IR), mediante la cancelación de una cuota mensual preestablecida y fija (Días \& Pacheco, 2013); siendo que en el cantón Cuenca, según datos presentados en la página web del SRI (2019) existen 21.400 contribuyentes pertenecientes al RISE, quienes representan el $20.38 \%$ del total de contribuyentes registrados. Es así que, en cuanto a la ciudad de Cuenca concretamente en la Parroquia el Vecino según la caracterización realizada a su contribuyentes por parte de los autores Piedra, Salinas y Vazques (2016) se evidenció que el $51 \%$ de éstos pertenecen al RISE, lo cual da cuenta de la importancia de éste Régimen y el grado de aceptación del mismo por parte de los contribuyentes de la ciudad.

Por otro lado, según el Plan Estratégico Institucional 2016-2019 del SRI (2016), en su objetivo de incrementar el cumplimiento voluntario de los impuestos se plantea consolidar una Institución más cercana con el contribuyente, bien sea de forma presencial, así como de manera virtual, desarrollado diferentes servicios en vías de facilitar el cumplimento de la obligaciones, mediante la estrategia de "automatizar y virtualizar los servicios de asistencia a los ciudadanos con un enfoque de simplicidad"; aunque es importante enmarcar éstos propósitos en la realidad ecuatoriana, donde el acceso a internet en el año 2015 llegó al 48.94\% superando al promedio mundial (43.99\%) y por debajo del Latinoamericano (54.55\%), siendo explicado este fenómeno por el alto analfabetismo digital que se ve incrementado en los sectores minoritarios y marginados de la sociedad (Vaca \& Merchán, 2017), lo cual constituiría un obstáculo para la imprescindible simplicidad tributaria.

Por lo expuesto, el presente trabajo investigativo tiene como objetivo caracterizar a los comerciantes de dulces de la festividad del Corpus Christi en la ciudad de Cuenca del año 2018, en su condición de contribuyentes, visibilizando sus percepciones y actitudes frente a las facilidades que presenta el SRI para el cumplimiento de las obligaciones tributarias; con lo cual, se pretende generar una base para futuras investigaciones en un sector poco estudiado y constituirse como un aporte para la toma de decisiones por parte de los entes coordinadores de la festividad y los entes regentes de los tributos en el país, en aras del cumplimiento del principio de simplicidad administrativa.

Como estructura de este documento se puede anotar que, en primer lugar, luego de este apartado introductorio, se 
presentan algunos conceptos y puntualizaciones identificados en la revisión de la literatura pertinente al caso, seguido de los aspectos metodológicos en los que se enmarcó el estudio planteado, posterior a lo cual se exponen los principales resultados de la investigación, en base a los cuales se exponen, en la parte final, las conclusiones del caso.

\section{Revisión de literatura}

Según se anotó en los párrafos introductorios de esta exposición, un elemento importante de las festividades tradicionales, en la ciudad de Cuenca, son sus comidas típicas, siendo infaltables en la celebración del Corpus Christi los dulces típicos como los quesitos, cocadas, suspiros, rosca enconfitada y otros más, los cuales son vendidos, en algunos casos por sus propios productores o por comerciantes, durante los días del festejo. Montoya (2010) reconoce en su investigación la importancia de las tradiciones alimentarias como elemento importante en la construcción de la identidad de los pueblos, su seguridad alimentaria y la relación con el ambiente natural, las cuales se sustentan en la transmisión del conocimiento de una generación a otra. Es así que, generalmente, al ser el conocimiento de lo tradicional transmitido de manera informal, según los datos de Chuguevskaia (2017) para el caso de los Evenks en Rusia, éste tiende a ser amenazado por dos factores como son la globalización y la modernización de los estilos de vida, lo que desencadena en un distanciamiento intergeneracional y una desvanecimiento de los saberes ancestrales.
Se reconocen otros casos de festividades tradicionales similares al Corpus Christi de la ciudad de Cuenca, tales como el de la Fiesta de la Primavera y la de Moros y Cristianos en España y las fiestas de Serfipe en Brasil donde se caracteriza a estos eventos a más de una expresión de la cultura de un pueblo, también como generadores de un auge en el volumen de actividad económica, con impactos que sobrepasan los límites locales (Palma, Palma, \& Martín, 2014; Perles, 2006; Santana, Lima, \& Possas, 2014).

Por lo expuesto, los comerciantes de dulces, vistos como emprendimientos productivos, se subordinan al régimen tributario ecuatoriano, el cual propende a una mayor eficiencia a fin de generar los recursos suficientes y oportunos que sostengan el gasto público, gestionados por el SRI, obteniendo resultados positivos, según la investigación de Ramírez y Carrillo (2017), quienes evidencian un repunte de la eficiencia recaudatoria del Impuesto al Valor Agregado (IVA) desde el 2007 y un crecimiento sostenido del Impuesto a la Renta IR en el período 2000-2014, sustentado en un mayor control por parte del ente y el fortalecimiento de la cultura tributaria en los contribuyentes.

Cabe subrayar que diversos autores definen a la cultura tributaria como una serie de conocimientos, actitudes y creencias, que son comunes a una sociedad en referencia al pago de los impuestos (Largo, Torres, y Moreno, 2019; Castillo y Castillo, 2016; Santillán, 2019). Es así como, Onofre, Aguirre y 
Murillo (2017) identifican, en los contribuyentes del Cantón Babahoyo, una relación directa entre la información sobre los tributos y su cumplimiento. De la misma manera se identificó para el caso de las personas naturales de la Provincia de Santa Elena que un $40 \%$ no cumple sus obligaciones tributarias por desconocimiento y un $54 \%$ por desinterés (Arriaga, Reyes, Olives, \& Méndez, 2017).

Finalmente, cabe destacar los esfuerzos del SRI a fin de simplificar los procesos inherentes al cumplimiento tributario, principalmente por el proceso de virtualización de sus servicios, por lo que según se destaca en su página web actualmente están disponibles 81 servicios en línea, que van desde la actualización de Registro Único de Contribuyentes (RUC), declaración y solicitud de devolución de distintos impuestos, obtención de certificados, pagos y otros (SRI, s/f); más, Paredes (2016) en su estudio en la ciudad de Guayaquil reconoce que el $47.8 \%$ de la muestra no considera que las declaraciones por internet facilitan su cumplimiento y tan solo el $21.4 \%$ de los estudiados reciben información de los tributos vía en línea.

\section{Metodología}

Esta investigación se basa en el método inductivo, mediante el cual el autor reconoce las particularidades en un caso específico, con el objetivo de contribuir a la definición de un fenómeno social. Se cataloga a la investigación planteada, como del tipo exploratorio, la cual se desarrolla con el fin de evidenciar los fundamentos de una problemática concreta muy poco estudiada, determinando aspectos claves que servirán de base para futuras investigaciones. De la misma manera se puntualiza que el presente trabajo investigativo se define como del tipo no experimental y con corte transversal; dado que se estudió el comportamiento de una muestra concreta de una población dada, en un momento específico, en su estado natural.

Se delimita como la población del presente estudio a los 113 comerciantes de dulces que asistieron a la festividad del Corpus Christi del año 2018, según datos proporcionados por la Dirección de Áreas Históricas y Patrimoniales del GAD Municipal del Cantón Cuenca 2015-2018 (2018); por lo que se decidió trabajar con una muestra definida en 59 comerciantes, la cual representa un nivel de confianza del $92 \%$, una proporción del $50 \%$ y un margen de error del $8 \%$; siendo que, para su conformación, se aplicó un muestreo de tipo aleatorio, donde se escogió al azar los comerciantes participantes del festejo a los que se aplicó el instrumento de investigación,

Por otro lado, se especifica que en el trabajo se utilizó una encuesta como herramienta investigativa, la que se aplicó en los diferentes días de la festividad y se estructuró de tal manera que se logre una adecuada caracterización de los contribuyentes y de su percepción sobre la simplicidad de los procedimientos.

En primera instancia, para el análisis 
de los datos se empleó estadísticos descriptivos y tablas de frecuencia; posteriormente al ser cualitativas el mayor número de variables, se empleó la prueba de Chi-cuadrado a fin de evaluar el grado de independencia de las variables tanto del tipo ordinal como nominal, dado el caso que se estudia. Finalmente, para evidenciar la existencia y el tipo de correlación entre las variables planteadas se empleó el Coeficiente de Spearman.

\section{Resultados}

Como punto de partida de la caracterización de los comerciantes de dulces que participaron en la festividad del Corpus Christi del año 2018 de la ciudad de Cuenca en su calidad de contribuyentes, y en base a la información proporcionada por la Dirección de Áreas Históricas y Patrimoniales del Gobierno Autónomo Descentralizado Municipal del Cantón Cuenca 2015-2018 (2018), se anota como datos, con la finalidad de tener una idea del perfil socio económico de los comerciantes, que el $37 \%$ de éstos son poseedores de vivienda propia y únicamente el $32 \%$ cuenta con afiliación a la seguridad social, dando cuenta de que los participantes en su mayoría son emprendedores con capacidad reducida para invertir en sus negocios y ven en la festividad una oportunidad para generar, de forma independiente, un ingreso para sus familias. Igualmente, se reconoce que la mayor proporción (96\%) de los comerciantes son del sexo femenino. De igual manera, 24 comerciantes, los cuales representan el $21 \%$ del total de los asistentes a la festividad elaboran los productos que expende; siendo que este grupo se conforma de 23 mujeres y un hombre; se anota que, el $79 \%$ de los vendedores que participan en el evento sólo comercializan los productos.

En base a lo anotado y las puntualizaciones que hace la Fundación Municipal de Turismo de Cuenca (2015), se verifica que desde sus orígenes la tradición de la elaboración de los dulces de la festividad del Corpus Christi recae sobre las mujeres de la ciudad, quienes son las portadoras del conocimiento que se ha transmitido por generaciones hasta el día de hoy.

Al analizar los datos referentes a las edades del total de los comerciantes, mediante el uso de la prueba de Kolmogorov-Smirnov, se obtuvo un valor -p menor a 0,05 no rechazando la hipótesis de que los datos de la variable siguen una distribución normal; reconociendo una edad promedio aproximada de 54 años en los participantes, con una desviación típica de 14.40 años, según se muestra en la Figura 1. Sumado a lo precedente, se evidenció una media de 62 años en las edades de los 24 comerciantes que son productores de los dulces, lo cual da cuenta de una falta de continuidad en la tradición, pudiéndose considerar, para el caso, como factor explicativo el distanciamiento intergeneracional según los explica Chuguevskaia (2017), lo cual ocasionaría una pérdida del conocimiento inherente a la preparación de los tradicionales bocadillos, sumado al hecho de que, en el mayor de los casos, dichos saberes se han venido 
transmitiendo de manera informal.

Por otro lado, se reconoce, que en su mayor porcentaje $(73 \%)$, los comerciantes que participan en la festividad se registran como contribuyentes en el RISE; siendo que, un $12 \%$ lo hace en el Régimen General y un $15 \%$ no está registrado como contribuyente (véase Figura 2); con lo cual, se verifica el alto grado de aceptación que tiene este modelo tributario, siendo este resultado consistente en relación con otras investigaciones citadas.

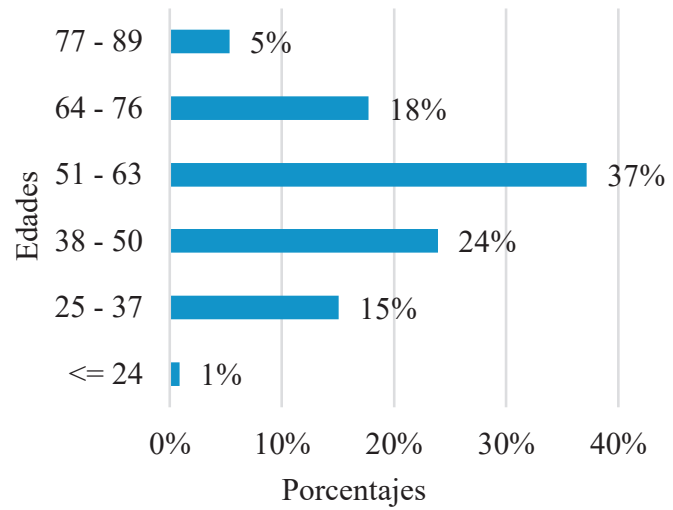

Figura 1. Clasificación de los comerciantes según su edad. Fuente: Dirección de Áreas Históricas y Patrimoniales del GAD Municipal del Cantón Cuenca 2015-2018 (2018).

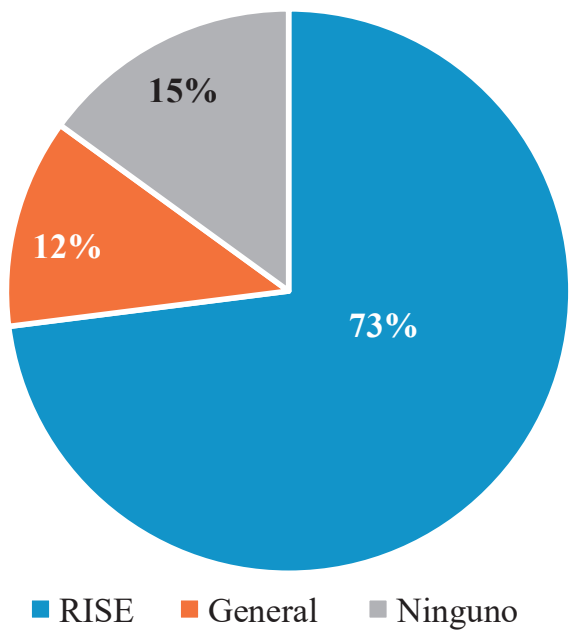

Figura 2. Clasificación de los comerciantes según el tipo de contribuyente.

Fuente: Dirección de Áreas Históricas y Patrimoniales del GAD Municipal del Cantón Cuenca 2015-2018 (2018).
Al evaluar la posible relación entre las variables tipo de contribuyente y su edad, mediante la Prueba de Chi-cuadrado, se confirma la existencia de una relación, estadísticamente significativa, entre las dos variables, según se presentan los datos en la Tabla 1. De igual forma, considerando al tipo de contribuyente como una variable ordinal, en donde 0 representan los asistentes que no cuentan con RUC, 1 los pertenecientes al RISE y 2 los que del Régimen General, se evaluó su asociación frente a la edad de los comerciantes, mediante el uso del Coeficiente de Correlación de Sperman; identificando una relación lineal, estadísticamente significativa, moderada e indirectamente proporcional entre las dos variables planteadas $(\mathrm{rho}=-0.449$, $\mathrm{p}<0.001$ ); por lo que se anota que los comerciantes de mayor edad serían los que tienden al incumplimiento tributario y los más jóvenes optan por el Régimen General.

Tabla 1.

Pruebas de Chi-cuadrado: edad-clase de contribuyente

\begin{tabular}{lccc}
\hline & Valor & gl & $\begin{array}{c}\text { Sig. asintótica } \\
\text { (bilateral) }\end{array}$ \\
\hline Chi-cuadrado de Pearson & $138,010^{\mathrm{a}}$ & 96 & 0,003 \\
Razón de verosimilitudes & 121,775 & 96 & 0,039 \\
Asociación lineal por lineal & 22,567 & 1 & 0,000 \\
\hline N de casos válidos & 113 & & \\
\hline
\end{tabular}

Fuente: Elaboración propia en base a los datos de Dirección de Áreas Históricas y Patrimoniales del GAD Municipal del Cantón Cuenca 2015-2018 (2018).

En cuanto al medio por el cual los comerciantes conocieron los requisitos necesarios para participar en el evento, se reconoce que en su gran mayoría $(93.22 \%)$ lo hicieron por medio de la ventanilla de información del Municipio de Cuenca; siendo que, únicamente, el $3.39 \%$ los conocieron por medios 
digitales. El resultado presentado se explicaría, según los datos presentados por Vaca y Merchán (2017), por la falta de acceso al internet en determinados grupos minoritarios, como es el caso de las personas adultas en el presente caso de estudio. Es así que se confirman los resultados obtenidos por Onofre, Aguirre y Murillo (2017), quienes concluyen que el acceso a la información es un factor que incide directamente en el cumplimiento de los tributos, por lo que se explicaría el incumplimiento de las personas adultas debido a un desconocimiento de sus deberes, dado a que la información se difunde principalmente por internet, la cual es recibida de forma limitada en algunos casos, según estudios previos (Paredes, 2016)

Por otro lado, al aplicar el instrumento de investigación, se evidencia que al clasificar a los comerciantes en su calidad de contribuyentes un $22.03 \%$ de los comerciantes encuestados no mantienen una actividad comercial habitual, siendo que obtuvieron su Registro Único de Contribuyentes (RUC) exclusivamente para participar como vendedor en la festividad y de este grupo el $92.31 \%$ prefirió regularizar su actividad mediante el RISE. De la misma manera, se destaca que el $37.29 \%$ ha mantenido el Registro por más de tres años y finalmente, se verifica que el $11.86 \%$ de la muestra del presente estudio participa como vendedor en la festividad incumpliendo la obligación tributaria de obtener su RUC.

Al realizar la evaluación de la independencia de las variables clase de contribuyente y tiempo que tiene el RUC mediante la prueba de Chi Cuadrado, según refieren los resultados presentados en la Tabla 2, se obtuvo un valor del estadístico de 63.22 y una significancia menor a 0.05 ; siendo que se rechaza la hipótesis nula y se confirma estadísticamente la relación de dependencia entre las variables.

Tabla 2.

Pruebas de Chi-cuadrado: edad-clase de contribuyente tiempo que tienen el RUC

\begin{tabular}{llcl}
\hline & Valor & gl & $\begin{array}{c}\text { Sig. asintótica } \\
\text { (bilateral) }\end{array}$ \\
\hline Chi-cuadrado de Pearson & $63,222^{\mathrm{a}}$ & 10 & 0,000 \\
Razón de verosimilitudes & 47,985 & 10 & 0,000 \\
Asociación lineal por lineal & 16,405 & 1 & 0,000 \\
\hline N de casos válidos & 59 & & \\
\hline
\end{tabular}

Fuente: Elaboración propia en base a los resultados de la encuesta de la investigación.

De la misma manera, al evaluar la correlación entre las dos variables planteadas, y considerándolas del tipo ordinales, mediante el análisis del coeficiente de correlación de Sperman, se reconoce una relación estadísticamente significativa $y$ positiva $\quad(\mathrm{rho}=0.375$, $\mathrm{p}<0.01$ ); es decir que, a más años como contribuyentes, los comerciantes prefieren el Régimen Impositivo General.

En línea con lo anotado, se identificó que los comerciantes de dulces han asistido en promedio 22 ocasiones a la celebración; siendo que, no existe una distribución clara de los datos, destacándose rangos como el de 33 a 40 ocasiones que asisten al evento con una proporción del $20 \%$ de comerciantes y otro $29 \%$ que afirma participará entre 9 y 16 oportunidades, dentro de los más importantes. Se reconoció que existe un porcentaje del $8 \%$ que ha participado en 41 ocasiones o más, según se presentan 
los resultados en la Figura 3.

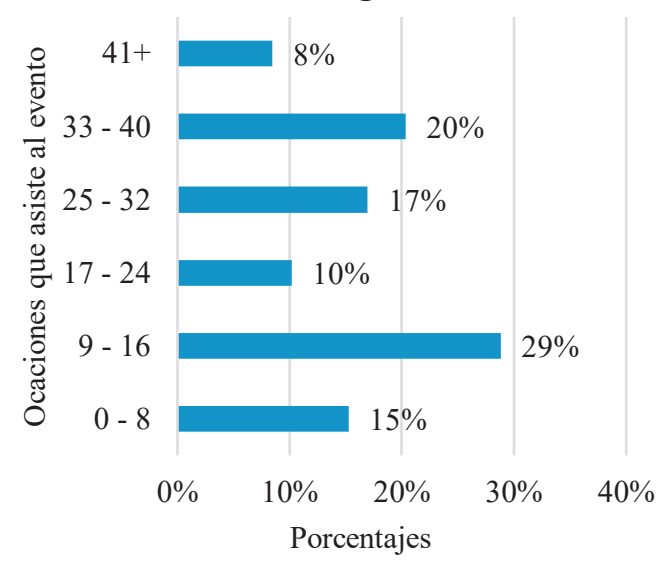

Figura 3. Ocasiones que asiste el comerciante a la festividad. Fuente: Resultados de la encuesta aplicada a los comerciantes.

Al evaluar la relación entre las variables tiempo que tienen el RUC y el número de ocasiones que participa en el evento, a la luz de los resultados de la Prueba de Chi-cuadrado de Pearson, se acepta la hipótesis de independencia, siendo que no existe evidencia estadística de una relación entre las variables planteadas.

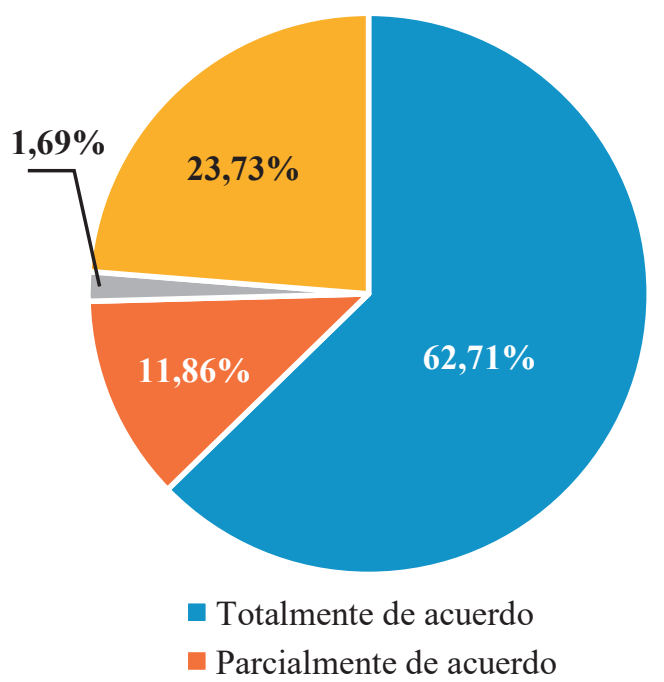

Figura 4. Considera simple cumplir con los requisitos solicitados.

Fuente: Resultados de la encuesta aplicada a los comerciantes de dulces de la festividad del Corpus Christi, Cuenca-2018.

De igual forma, se advierte que, aunque el $62.71 \%$ de los consultados considera estar totalmente de acuerdo con la afirmación de que es sencillo cumplir con los requisitos tributarios requeridos para regularizar su participación como comerciante en la festividad (ver Figura 4), y tomando en cuenta los datos presentados, las personas de mayor edad acceden menos al internet y son las que recaen en el incumplimiento tributario, pudiéndose explicar el fenómeno por el desconocimiento de sus obligaciones, visto el caso ya presentado de los contribuyentes de la Provincia de Santa Elena (Arriaga, Reyes, Olives, \& Méndez, 2017).

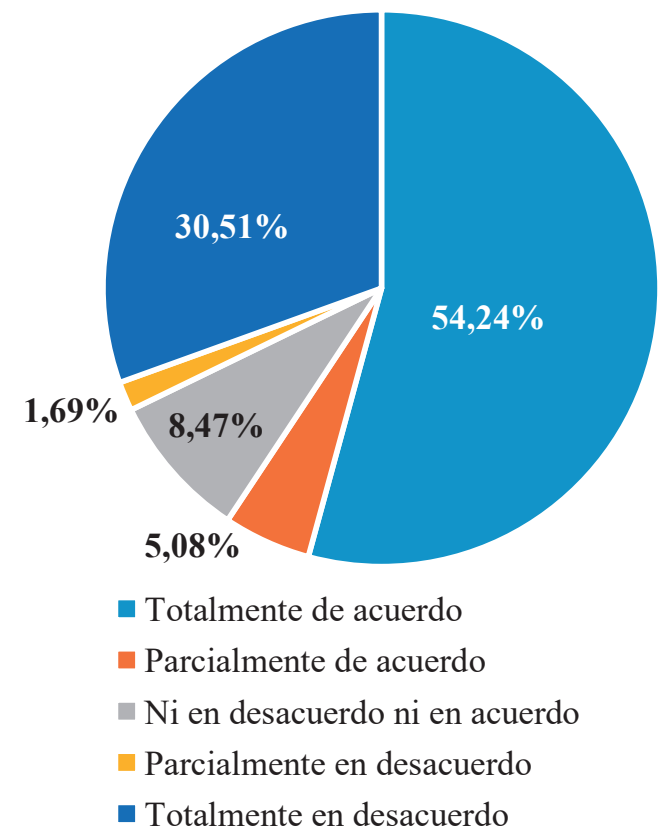

Figura 5. Las TIC's favorecen el cumplimiento de las obligaciones tributarias.

Fuente: Resultados de la encuesta aplicada a los comerciantes de dulces de la festividad del Corpus Christi, Cuenca-2018.

Considerando el proceso de virtualización de muchos procesos inherentes al cumplimiento tributario por parte del SRI, se reconoce, en la presente investigación, que un alto porcentaje de los encuestados (54.24\%), según se aprecia en la Figura 5, consideran estar de 
acuerdo con la afirmación de que las TIC's favorecen el cumplimiento de los requisitos necesarios para participar en calidad de comerciantes en el evento; sin embargo, el $30.51 \%$ está en desacuerdo con la afirmación planteada. Estos resultados son concordantes con los obtenidos en investigaciones previas, donde altos porcentajes de las muestras estudiadas no considera al internet como un factor facilitador en el cumplimiento tributario (Paredes, 2016).

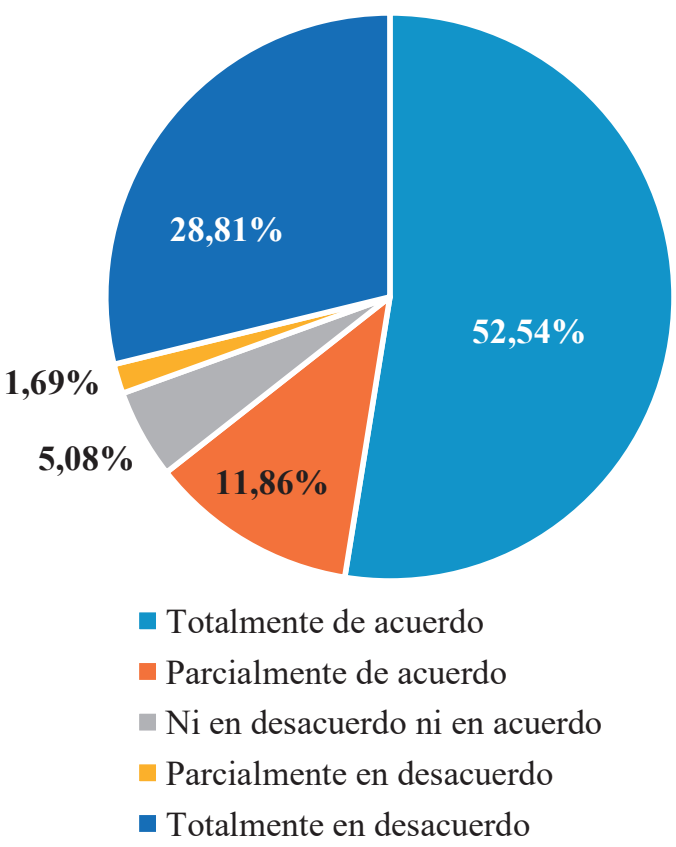

Figura 6. Necesidad de apoyo para cumplir con los requisitos establecidos.

Fuente: Resultados de la encuesta aplicada a los comerciantes de dulces de la festividad del Corpus Christi, Cuenca-2018.

Al evaluar el grado de autonomía que tienen los comerciantes para cumplir con los requisitos tributarios solicitados en su calidad de comerciantes asistentes a la festividad del Corpus Christi, según los resultados que se aprecian en la Figura 6, en su mayor porcentaje $(64.40 \%)$ están de acuerdo o totalmente de acuerdo con el planteamiento de que tuvieron la necesidad de apoyo para cumplir con los requisitos tributarios establecidos para asistir al evento; mientras que, un 30.50\% de los encuestados fueron autónomos en cuanto a su capacidad de cumplir con los requerimientos trazados, dada su posición tanto de desacuerdo como totalmente en desacuerdo con el enunciado sugerido.

\section{Conclusiones}

Luego de la investigación propuesta, en primera instancia se puntualiza que la festividad del Corpus Christi constituye un acto de relevante significancia cultural que ha sido preservado en la ciudad de Cuenca como parte de su patrimonio intangible; el cual se constituye como un espacio para que la tradición de la elaboración y venta de dulces, propios de la fiesta, se conserve y a la vez genere una oportunidad para que pequeños emprendedores desarrollen una actividad comercial con los réditos pertinentes.

En atención al objetivo de caracterizar a los comerciantes de dulces de la festividad del Corpus Christi en la ciudad de Cuenca del año 2018, se confirma que desde sus orígenes hasta la actualidad han sido las personas del sexo femenino las portadoras de conocimiento inherente a la fabricación de los dulces y las que actualmente se encargan, en mayor proporción, tanto de su fabricación y expendio, durante los días que dura el festejo. De la misma manera, se pone en evidencia que existe una tendencia a que los conocimientos inherentes a la elaboración de los dulces tradicionales desaparezcan, dada la avanzada edad de 
las productoras; siendo importante que en investigaciones posteriores se identifiquen las barreras que obstaculizan el relevo generacional, el cual afectaría la conservación de la tradición estudiada.

En línea con lo expuesto, se evidencia que un alto porcentaje de comerciantes no tiene como actividad habitual la venta y más aún la producción de los dulces; pues, se registraron como contribuyentes, únicamente, para participar en el evento; aunque, se identificó un número alto de ocasiones que han asistido a la festividad; con lo cual, se confirmaría el hecho de que cada vez son menos los productores, y el evento concentra cada vez más a comerciantes, con lo que se estaría reduciendo la oferta permanente de los tradicionales dulces en la ciudad de Cuenca.

Por otra parte, en referencia a las percepciones $\mathrm{y}$ actitudes frente a las facilidades que presenta el SRI para el cumplimiento de las obligaciones tributarias, se confirma el interés que suscita el RISE, dadas sus particularidades, como un modelo de tributación accesible para los pequeños emprendedores; siendo que, se pone en evidencia que los contribuyentes más jóvenes y los que únicamente se registran para el evento, prefieren este modelo dada su accesibilidad. Igualmente se establece que los contribuyentes de mayor edad son más propensos a caer en incumplimiento de sus obligaciones tributarias, debido principalmente al limitado acceso a la información que se difunde por medios digitales, siendo que en su mayoría la reciben de forma directa en las ventanillas de la Municipalidad.

El limitado acceso a los medios digitales por parte del grupo de estudio, constituido éste por personas que en su mayoría superan los cincuenta años, se confirma dado que se evidenció que un porcentaje considerable piensa estar en desacuerdo con la afirmación de que las TIC's favorecen el cumplimiento de las obligaciones tributarias, siendo que se advierte una baja autonomía por parte de los comerciantes para cumplir con los requisitos establecidos para su asistencia al evento. Es así como se podría afirmar que el uso de las tecnologías constituye una barrera para el cumplimiento tributario para las personas de edades avanzadas; siendo que el uso de las TIC's favorecen el principio de simplicidad únicamente en el caso de los contribuyentes considerados jóvenes o actualizados en los temas tecnológicos.

De la misma manera, no se estableció una relación estadísticamente significativa entre el tipo de contribuyente y el número de ocasiones que los comerciantes asisten al evento, con lo cual se concluye que los años de experiencia como contribuyente no determina el tipo de este. Finalmente, cabe anotar que con el trabajo presentado se pretende dar respuesta a la pregunta de investigación planteada, pues se considera oportuno visibilizar las percepciones y actitudes de un sector específico frente a sus obligaciones tributarias, por lo que al reconocer este trabajo como del tipo exploratorio en un sector concreto de la economía, será importante, en futuras investigaciones, profundizar el estudio de la relación entre 
el uso de las herramientas informáticas y el cumplimiento tributario.

\section{Referencias}

Arriaga, G., Reyes, M., Olives, J., y Méndez, V. (2017). Análisis de la cultura tributaria: impuesto a la renta para persona naturales no obligadas allevar contabilidad, Provincia de Santa Elena. Revista de Ciencias Pedagógics e Innovación(3), 118-127.

http://dx.doi.org/10.26423/rcpi.v5i3.192

Asamblea Constituyente. (2008). Constitución de la República del Ecuador. Registro Oficial No. 449.

Astudillo, L. (2011). Estudio de caso: El septenario cuencano. En F. d. Porto, El pensamiento museológico contemporáneo (págs. 89-97). Buenos Aires: Comité Internacional del ICOM para la Museología.

Bermejo, A. (2012). La máscara del diablo, como elemento simbólico, en la celebración del Corpus Christi (Atánquez-Colombia). Boletín Antropológico, 30(83), 73-103. https://www.redalyc.org/pdf/712/712254 38004.pdf

Castillo, S., \& Castillo, P. (2016). Un acercamiento al estudio de la cultura tributaria desde la perspectiva socioeducativa. Dominio de las Ciencias, 2(3), 149-162.

http://192.188.49.30/index.php/suracade $\mathrm{mia} /$ article/view/521

Chuguevskaia, A. (2017). Stepanovna. The Loss of Oral Traditions in the Far East Russia: Future of the Community. Geconservación, 11, 149-156.

https://www.ge-iic.com/ojs/index.php/rev ista/article/view/467

Congreso Nacional. (1997). Ley de Creación del Servicio de Rentas Internas [Ley No. 41]. Registro Oficial No. 206, 2 de Diciembre de 1997.
http://www.sri.gob.ec/BibliotecaPortlet/d escargar/80a4f4ca-2d92-49b4-8854-d0df $50 \mathrm{e} 13 \mathrm{~b} 9 \mathrm{a} / 4 \% 29 \mathrm{LEY}+\mathrm{DE}+\mathrm{CREACIO} \%$ $\mathrm{B} 4 \mathrm{~N}+\mathrm{DEL}+\mathrm{SERVICIO}+\mathrm{DE}+\mathrm{RENTAS}+$ INTERNAS.pdf

Congreso Nacional. (2005). Código Tributario. Registro Oficial Suplemento $38 \mathrm{de}$ 14-jun.-2005.

Días, J., \& Pacheco, F. (2013). El régimen impositivo simplificado ecuatoriano Rise desenvolvimiento y perspectivas. ECA Sinergia, 4(1), 30-37. https://dialnet.unirioja.es/servlet/articulo ?codigo $=6197550$

Dirección de Áreas Históricas y Patrimoniales del GAD Municipal del Cantón Cuenca 2015-2018. (2018). Base de datos Corpus Christi. [Archivo de Excel]. Archivos de la Dirección General de Áreas Históricas y Patrimoniales.

Fundación Municipal de Turismo de Cuenca. (2015) La fiesta del Corpus Christi en Cuenca. https://issuu.com/funturismo/docs/folleto corpusrojo

Largo, N., Torres, D., \& Moreno, Y. (2019). Cultura tributaria de las MIPYMES y su incidencia en el contexto socio económico ecuatoriano. Sur Academia: Revista Académica-Investigativa de la Facultad Jurídica, Social y Administrativa, 4(8). http://192.188.49.30/ index.php/suracademia/article/view/521

Ministerio de Economía y Finanzas. (2018). Proforma del Presupuesto General del Estado Reporte Consolidado Comparativo por Grupo 2019.

https://www.finanzas.gob.ec/wp-content/ uploads/downloads/2018/12/4-CN_PorGrupo_Ingresos.pdf

Montoya, F. (2010). Tradiciones alimentarias: bienestar de las personas y el ambiente. Cuadernos de Antropología, 20(1). https://dialnet.unirioja.es/descarga/articul 
o/6066267.pdf

Onofre, R., Aguirre, C., \& Murillo, K. (2017). La cultura tributaria y su incidencia en la recaudación de los tributos en el Cantón Babahoyo, Provincia de los Ríos. Dominio de las ciencias, 3(3), 3-23. http://dx.doi.org/10.23857/dom.cien.poca ip.2017.3.3.jun. $45-68$

Palma, L., Palma, M., \& Martín, J. (2014). La integración entre cultura y economía. El caso de las Fiestas de Primavera de Sevilla. Estudios de Economía Aplicada [en linea] 2014, 32(1).

http://www.redalyc.org/articulo.oa? id=30 129510014> ISSN 1133-3197

Paredes, P. (2016). Evasión tributaria vs. mecanismos de control implementados por la administración pública. Retos, 6(12), 180-198.

https://doi.org/10.17163/ret.n12.2016.04

Perles, J. (2006). Análisis del impacto económico de eventos: una aplicación a fiestas populares de proyección turística. Cuadernos de Turismo(17), 147-166. http://www.redalyc.org/pdf/398/3980170 7.pdf

Piedra, A., Salinas, J., \& Vazques, J. (2016). Caracterización tributaria en barrios urbanos populares: Caso parroquia El Vecino. RETOS, 6(11), 88-104. doi:10.17163/ret.n11.2016.06

Prada, J., Armijos, D., Creso, A., \& Torres, L. (2018). El turista cultural: tipologías y análisis de las valoraciones del destino a partir del caso de estudio de Cuenca - Ecuador. Pasos, 16(1), 55-72.

https://doi.org/10.25145/j.pasos.2018.16. 004

Ramírez, J., y Carrillo, P. (2017). Indicador de Eficiencia de la Recaudación del Impuesto al Valor Agregado y del Impuesto a la Renta de Ecuador. Documento de trabajo del BID; 852. https://papers.ssrn.com/sol3/papers.cfm? abstract_id $=2604162$

Santana, L., Lima, J., y Possas, G. (2014). Impactos económicos de los gastos turísticos en Sergipe y sus efectos colaterales en el resto de Brasil. Estudios y Perspectivas en Turismo, 23(3), 447-446.

Santillán, M. (2019). La cultura tributaria en el sector artesanal y su efecto en el cumplimiento de obligaciones. Visión Empresarial(9), 41-50.

https://doi.org/10.32645/13906852.868

SRI. (2016). Plan Estratégico Institucional 20162019. https://www.sri.gob.ec/Documentos AlfrescoPortlet/descargar/3828605d-f9a1 -481a-b23e-6d18bfdefab3/PLAN+ESTR AT\%C9GICO+INSTITUCIONAL +2016 -2019.pdf.

SRI. (2019). Catastros. https://www.sri.gob.ec/web/ guest/catastros

SRI. (s/f). Servicios en línea. https://www.sri.gob.ec/ web/guest/servicios-en-linea5

Vaca, M., \& Merchán, C. (2017). Tecnologías de información y comunicaciones: evolución y brecha digital en Ecuador. Revista Renavatio, 1(1). http://cienciaytecnologia.uteg.edu.ec/revi sta/index.php/renovatio/article/view/126

Viera y Clavijo, J. (2017). Reales academias. Santa Cruz de Tenerife, España: Ediciones idea. 
